\section{Vésicules extracellulaires et maladies cardiovasculaires}

Pierre-Michael Coly ${ }^{1}$, Xavier Loyer ${ }^{1}$

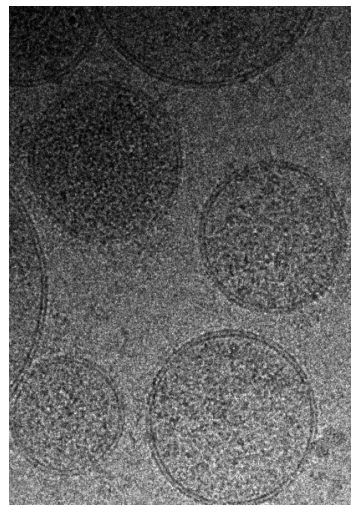

Université de Paris, Inserm UMR 970, Paris-Centre de recherche cardiovasculaire (Paris-Cardiovascular Research Center), 56 rue Leblanc, F-75015 Paris, France.

xavier.loyer@inserm.fr pierre-michael.coly@inserm.fr

plupart des types cellulaires. Les VE sont généralement classifiées en trois sous-catégories: 1) les exosomes (30-100 nm), qui sont formés lorsque les endosomes multivésiculaires fusionnent avec la membrane plasmique et libèrent leur contenu dans l'espace extracellulaire; 2) les microvésicules (100-1000 nm), issues d'un bourgeonnement de la membrane plasmique vers l'espace extracellulaire; et 3 ) les corps apoptotiques (1-5 $\mu \mathrm{m})$ qui, comme leur nom l'indique, sont libérés par des cellules mourantes. La Société internationale des vésicules extracellulaires (ISEV) a récemment proposé une nomenclature se fondant sur la caractérisation des VE selon leur taille, distinguant les grandes VE (ou microvésicules) et les petites VE (ou exosomes). Cette synthèse se référera au terme générique de $V \varepsilon$.

Les recherches actuelles s'intéressent à la capacité intrinsèque des $V \varepsilon$ à transporter des composés bioactifs tels que des acides nucléiques, des lipides et des protéines. En effet, la composition des VE est le reflet de l'état physiologique de leur cellule d'origine. De plus, les $V \varepsilon$ peuvent aussi modifier le phénotype des cellules qui les incorporent, en apportant leurs constituants [2]. Ainsi, de nombreuses études ont montré un rôle des $V \varepsilon$ dans différents processus physiologiques et pathologiques [3]. Ces dernières années, des efforts considérables ont été déployés afin d'élucider le rôle des VE dans l'initiation et la progression des maladies cardiovasculaires. Ces vésicules peuvent être libérées par tous les types cellulaires qui composent le système cardiovasculaire, tels que les cellules endothéliales, les cardiomyocytes, les cellules musculaires lisses, ainsi que les cellules sanguines.

Cette synthèse a pour but de discuter les études récentes évaluant le rôle des Vદ dans les maladies cardiovasculaires, principalement dans les contextes d'athérosclérose et d'infarctus du myocarde (IM). 


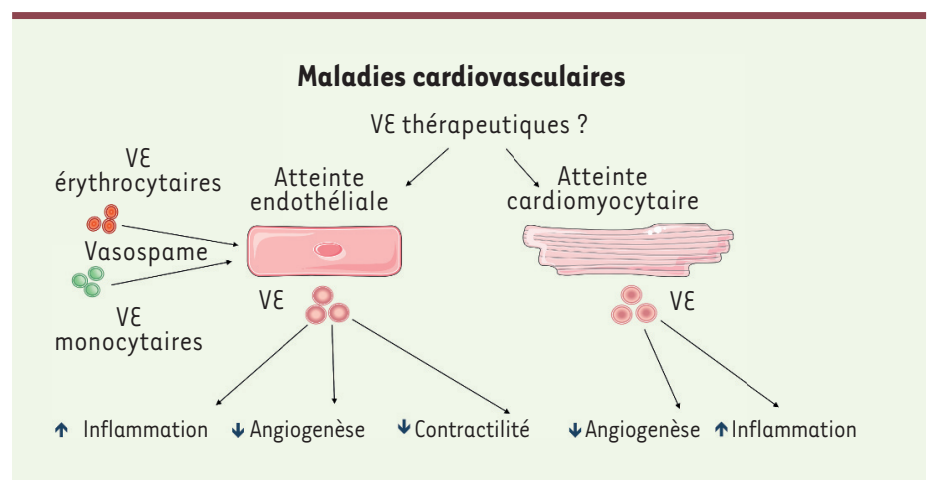

Figure 1. Représentation schématique du rôle des vésicules extracellulaires (VE) dans les maladies cardiovasculaires. Dans certains contextes, des $V \varepsilon$ circulantes de différentes origines (érythrocyte, monocyte, plaquette, etc.) contribuent au dysfonctionnement endothélial. Les VE libérées à la suite d'une atteinte endothéliale peuvent moduler l'angiogenèse, l'inflammation et la contractilité vasculaire, notamment via le transfert intercellulaire de microARN, de cytokines et de protéines membranaires. En réponse à une ischémie, le tissu myocardique lésé libère des $V \varepsilon$ dans la circulation. Ces $V \varepsilon$ influencent les processus d'angiogenèse et d'inflammation en interagissant avec des cellules endothéliales ou avec des cellules inflammatoires infiltrant le tissu. Tirer parti des effets bénéfiques de certaines sous-catégories de $V \varepsilon$ pourrait permettre à de nouvelles stratégies thérapeutiques de voir le jour.

\section{Vésicules extracellulaires et maladies vasculaires}

\section{Vع et athérosclérose}

Du stade initial aux lésions plus avancées [4], les plaques d'athérosclérose contiennent des vésicules extracellulaires, suggérant que celles-ci pourraient jouer un rôle au cours du développement de la maladie. Plusieurs travaux ont montré par ailleurs que les niveaux circulants de VE dérivées de plaquettes et de cellules endothéliales étaient significativement augmentés chez des patients souffrant d'athérosclérose [5], tandis que les niveaux circulants de VE leucocytaires sont associés à des plaques plus instables chez des patients asymptomatiques [6]. II faut souligner que la présence de VE endothéliales est plus fortement corrélée avec la progression et la sévérité de la maladie que d'autres marqueurs plus classiques de l'activation endothéliale, tels que la protéine ICAM-1 (intercellular adhesion molecule-1) [7]. Elles apparaissent donc comme des candidats prometteurs pour prédire la survenue d'évènements cardiovasculaires chez des patients à risque [8].

Plusieurs mécanismes ont été proposés pour expliquer le rôle des VE au cours de la formation des plaques d'athérosclérose. Des données obtenues in vitro indiquent que les $V E$ de différentes origines peuvent stimuler la libération de cytokines pro-inflammatoires par les cellules endothéliales et les leucocytes, comme les interleukines (IL) 6 et 8 [4]. Des $V E$ d'origine endothéliale, plaquettaire ou érythrocytaire peuvent également augmenter la perméabilité de l'endothélium et favoriser l'adhérence des leucocytes (Figure 1). De plus, des cellules endothé- liales exposées à des conditions pro-athérogènes (LDL [low density lipoprotein] oxydées, faibles contraintes de cisaillement, TNF- $\alpha$ [tumor necrosis factor alpha]) libèrent des VE transportant les micro-ARN-92 et 155, ainsi qu'un cocktail de cytokines et chimiokines ( $\mathrm{CCL}$ [C $-C$ motif chemokine ligand $] 2$ et $5, \mathrm{IL}-6$ et IL-8, CXCLIO [C-X-C motif chemokine ligand 10], TNF- $\alpha$ ) $[4,9]$. Une fois captées par des monocytes, ces $V \mathcal{E}$ sont capables de les orienter vers un phénotype pro-inflammatoire (ou type $\mathrm{Ml}$ ). Ces évènements permettent alors l'infiltration des monocytes dans l'endothélium, favorisant ainsi l'initiation de la maladie. Certains de ces effets peuvent être atténués par des traitements ciblant l'hypertension ou l'hyperlipidémie. Comparées à des $V \varepsilon$ provenant de patients hypertendus ou hyperlipidémiques, des $V \varepsilon$ isolées de patients traités peuvent en effet réduire in vitro l'expression des protéines VCAM-1 (vascular cell adhesion molecule 1) et ICAM-1 par des cellules endothéliales en culture (cellules HUVEC, pour human umbilical vein endothelial cells) [10].

Différents types de $V \varepsilon$ peuvent stimuler la transformation de macrophages en cellules spumeuses en favorisant l'accumulation de lipides au sein de ces cellules. C'est le cas, par exemple, des VE dérivées de lymphocytes T, capables de stimuler l'accumulation de cholestérol [11]. Ce processus mène généralement à une apoptose de ces macrophages qui peut être accélérée par le transfert des caspases 1 et 3 via des $V \varepsilon$ provenant de cellules endothéliales, d'érythrocytes, de plaquettes, de monocytes, ou de cellules dendritiques [4]. Les cellules musculaires lisses, au sein de la plaque d'athérosclérose, peuvent également être influencées par les $V \varepsilon$ environnantes. En effet, des $V \varepsilon$ plaquettaires contenant du PDGF (platelet-derived growth factor) stimulent la prolifération de ces cellules [12]. À l'inverse, leur apoptose peut être activée par des VE dérivées de monocytes transportant la caspase 1 [13]. Plusieurs études soulignent un rôle des VE dans la déstabilisation de la plaque d'athérome et dans la thrombose. Une caractérisation de plaques humaines vulnérables a montré que le noyau nécrotique de ces plaques contient de grandes quantités de $V E$ thrombogènes provenant principalement de leucocytes, d'érythrocytes et de cellules musculaires lisses [14, 15]. Cette abondance de vésicules particulières au sein de la plaque confère un haut potentiel pro-coagulant à la lésion athéroscléreuse, par rapport à des $V \varepsilon$ circulantes provenant du même patient [14]. La rupture de la chape fibreuse de la lésion expose donc ce matériel thrombogène, déclenchant ainsi une activation plaquettaire et la formation de thrombus au site de la lésion [4]. Les VE participent activement à l'initiation de la cascade de 
coagulation via le facteur tissulaire et la phosphatidylsérine qu'elles présentent à leur surface $[14,16]$. Les VE circulantes affectent également le processus de coagulation [4] et peuvent potentiellement initier celui-ci en se liant à la matrice subcellulaire à la suite d'une lésion endothéliale [17]. Alors que la plupart de ces effets ont été attribués aux microvésicules, certains types de petites $V \varepsilon$ semblent jouer un rôle anti-thrombotique. En effet, les petites VE dérivées de plaquettes ralentissent l'agrégation plaquettaire et préviennent la thrombose occlusive dans un modèle murin de lésion de l'artère carotide, en réduisant les niveaux d'expression de CD36 (ou fatty acid transporter, FAT) plaquettaire [18].

En plus de moduler la perméabilité de l'endothélium, les VE interviennent dans la dégradation de la matrice extracellulaire via les activités des métalloprotéases qu'elles abritent [19]. Des fuites peuvent ainsi être induites au sein des vasa vasorum ${ }^{1}$ immatures, favorisant le recrutement de cellules inflammatoires qui contribueront, par la suite, à la déstabilisation de la plaque [20]. Les processus néo-angiogéniques locaux sont également influencés par les $V \varepsilon$ de la plaque elles-mêmes (Figure 1). Cet effet repose sur l'interaction entre le CD40 endothélial et son ligand (CD40L), présent à la surface des vésicules dérivées des macrophages de la plaque ${ }^{2}$ [19]. L'activité fibrinolytique des VE leucocytaires et endothéliales pourrait également contribuer à ce processus angiogénique [4]. Les $V \varepsilon$ peuvent également influencer la dégradation des protéines structurelles par le biais des métalloprotéinases matricielles (MMP) qu'elles peuvent exprimer à leur surface. Les $V \varepsilon$ de macrophages, générées in vitro, expriment en effet la MMP-14 (ou MTl-MMP pour membrane-type matrix metalloproteinase 1) à la suite d'une exposition à la fumée de cigarette. De même, les VE endothéliales, générées in vivo et in vitro, expriment les MMP-2, -9, - 10 ou -14 et les VE dérivées de neutrophiles portent MMP-9 [4].

Un rôle potentiel de $V \varepsilon$ issues d'adipocytes a été montré dans l'athérosclérose. En effet, des VE d'origine adipocytaire favorisent l'adhérence de cellules inflammatoires aux cellules endothéliales, conduisant à la survenue d'athérosclérose [21]. Dans des modèles murins, ces $V \varepsilon$ altèrent la régulation de l'homéostasie du cholestérol des macrophages, favorisant ainsi la survenue de lésions athéromateuses. Enfin, il a été montré que ces $V \varepsilon$ issues d'adipocytes favorisaient la vulnérabilité des plaques d'athérosclérose et le développement de lésions d'athérosclérose en induisant l'angiogenèse des vasa vasorum, chez les souris diabétiques athéroprones [22].

\section{$V \varepsilon$ et altérations fonctionnelles des vaisseaux}

Les $V \varepsilon$ circulantes ont un impact sur les maladies cardiovasculaires, en altérant la synthèse de monoxyde d'azote (NO) au niveau endothélial, conduisant à une dysfonction endothéliale. Cette dysfonction des cellules endothéliales est associée à une augmentation de la production de radicaux libres de l'oxygène et à un stress oxydant intense entraînant des altérations de contractilité des vaisseaux, tels que des

1 Vaisseaux de très petit calibre cheminant dans l'adventice (tunique externe de la paroi d'une artère) et irriguant la paroi artérielle.

${ }^{2}$ CD40 est un corécepteur exprimé par le macrophage, induisant sa stimulation lorsque son ligand, CD40L, le fixe. vasospasmes. Ces vasospames sont associés à la survenue d'évènements cardiaques, sans obstruction des artères coronaires, dans des contextes pathologiques comme les néoplasmes myéloprolifératifs. Les néoplasmes myéloprolifératifs ont pour origine une mutation du gène qui code la kinase JAK2 (Janus kinase 2), la mutation JAK2 V617F conduisant à une activation constitutive du récepteur du facteur de croissance et des voies de signalisation en aval (indépendamment de ses ligands), ce qui aboutit finalement à la prolifération des cellules myéloïdes. Dans ce contexte pathologique, Poisson et al. ont montré un rôle des VE dérivées d'érythrocytes. En effet, les $V \varepsilon$ érythrocytaires portant la mutation JAK2 V617F sont transférées aux cellules endothéliales, où elles favorisent un stress oxydant en transmettant une enzyme, la myéloperoxydase (MPO) [23].

Un rôle des VE d'origine érythrocytaire a également été décrit dans le contexte de vasospames occlusifs associés à la drépanocytose. Dans ce contexte pathologique, les VE érythrocytaires de patients drépanocytaires présentent de fortes concentrations d'hème. Le transfert de l'hème aux cellules endothéliales, via les $V \varepsilon$ érythrocytaires, induit un stress oxydant important à l'origine d'une dysfonction endothéliale conduisant aux crises vaso-occlusives observées dans la maladie [24] (Figure 1).

\section{Vésicules extracellulaires (Vع) et atteinte myocardique}

En réponse à une ischémie, le tissu cardiaque lésé libère des $V \varepsilon$ dans la circulation. Les taux sériques de ces vésicules pro-coagulantes, de diverses origines (endothéliales ou dérivées des plaquettes), augmentent dans les heures qui suivent le début de l'ischémie myocardique. La surveillance de leurs taux chez les patients améliore ainsi les prédictions des événements cardiovasculaires indésirables majeurs [25]. L'utilisation de modèles murins d'ischémie cardiaque a révélé que les taux circulants de $V \varepsilon$ augmentent rapidement et que leur contenu est enrichi (contenu spécifique en micro-ARN d'origine cardiaque) [26]. Rodriguez et al. ont ainsi montré chez la souris, suite à un infarctus du myocarde, la présence dans la circulation de $V \varepsilon$ portant la connexine 43 , celle-ci représentant un marqueur potentiel d'origine cardiomyocytaire [27]. Chez l'homme, une récente étude a montré que le myocarde libère en continu des $V \varepsilon$ dans le sang. Cette libération est accrue en réponse à divers stress (sténose aortique ou affection des valves cardiaques) [28]. En utilisant une approche innovante de cytométrie en flux, Anselmo et al. ont mis en évidence la présence de $V \varepsilon$ exprimant 
à leur surface la protéine SIRP $\alpha$ (signal regulatory protein $\alpha$ ou CD172a), un marqueur des cardiomyocytes. L'origine cardiomyocytaire de ces VE exprimant CD172a (CD172a+) a été confirmée par la présence de troponine $T$, une protéine impliquée dans la contraction cardiaque, et leur enrichissement spécifique en micro-ARN cardiaques. Les taux de VE CD $172^{+}$constituent un marqueur de bon pronostic, un taux élevé étant associé à un pronostic favorable chez les patients. Cela est en partie dû à un transfert de VE CD $172^{+}$au tissu myocardique sain, ce qui favorise la contraction cardiaque par un maintien du cycle calcique.

Des VE d'origine endothéliale sont également retrouvées en grand nombre dans la circulation en réponse à un infarctus du myocarde [29]. Ces VE expriment à leur surface VCAM-1 et PECAM-1 (platelet endothelial cell adhesion molecule-1 ou CD31) et sont transférées dans la rate, où elles induisent la mobilisation de cellules inflammatoires circulantes (les monocytes) vers la zone ischémique lésée.

Dans le tissu ischémique, une libération intense et transitoire (dès $15 \mathrm{~h}$ après un infarctus du myocarde) de $V \varepsilon$ majoritairement d'origine cardiomyocytaire est observée. Ces $V \varepsilon$ vont ensuite être transférées aux cellules inflammatoires infiltrant le tissu, modulant alors la libération de cytokines pro-inflammatoires [30]. La présence de $V \varepsilon$ dans le tissu ischémique a été confirmée chez les patients. Une classe de VE spécifiques, les exophères, est libérée par les cardiomyocytes. Ces vésicules particulières contiennent des mitochondries dysfonctionnelles qui seront transférées, via un mécanisme impliquant la kinase MERTK (myeloid-epithelial-reproductive tyrosine kinase), aux macrophages cardiaques, qui les élimineront, participant ainsi au maintien de l'homéostasie cardiaque [31].

\section{Vésicules extracellulaires (VE) et cardiomyopathie diabétique}

La cardiomyopathie diabétique se définie comme un dysfonctionnement myocardique survenant chez des patients atteints de diabète, en l'absence de maladie coronarienne, d'hypertension ou de valvulopathie. Cette maladie comporte généralement un risque élevé d'insuffisance cardiaque et de mortalité [32].

De récents travaux ont montré que l'environnement diabétique est associé à des modifications significative des niveaux de VE circulantes, ainsi que de leur composition [33]. Des études in vivo portant sur le rôle des VE dans l'homéostasie cardiomyocytaire ont révélé que les VE dérivées d'adipocytes diabétiques, transportant le micro$A R N-130 b-3 p$, étaient transférées vers des cardiomyocytes lors d'une ischémie-reperfusion. En agissant sur des cibles telles que l'AMPK (AMP-activated protein kinase) $\alpha 1 / \alpha 2$, Birc6 (Baculoviral inhibitor of apoptosis repeat-containing protein 6) et Ucp3 (uncoupling protein 3), ce micro-ARN entraîne une exacerbation de la lésion, comme en témoignent la moindre récupération de la fonction cardiaque, ainsi que l'augmentation de l'apoptose cardiomyocytaire et de la taille de l'infarctus [34].

Au cours de l'évolution initiale du diabète, l'hyperglycémie peut entraîner des dysfonctionnements endothéliaux à l'origine d'une raréfaction microvasculaire au sein du myocarde [35]. II a d'ailleurs été suggéré qu'une dérégulation de l'angiogenèse myocardique serait la cause principale des cardiomyopathies diabétiques [36]. L'exposition à de fortes concentrations de glucose conduit à une élévation dans l'endothélium des niveaux du micro-ARN-503 qui, en ciblant les transcrits de la cycline Conel (cyclin 81 ) et de la phosphatase Cdc25A (cell division cycle $25 \mathrm{~A}$ ), inhibe la prolifération endothéliale et l'angiogenèse [37]. Le transfert du micro-ARN-503 via des $V \varepsilon$ endothéliales altère la migration et la prolifération des péricytes, réduisant davantage l'angiogenèse post-ischémique ainsi que la perméabilité vasculaire en interférant avec la production de VEGF-A (vascular endothelial growth factor $A$ ) et d'EFNB2 (ephrin B2) [38] (Figure 1). Dans des conditions physiologiques, les cellules endothéliales libèrent des VE enrichies en micro-ARN-10a, qui peut être ainsi transféré aux monocytes, où il réprime plusieurs acteurs de la voie de signalisation impliquant NF- $\kappa B$ (nuclear factor- $K B$ ), participant ainsi à l'atténuation de leur activité pro-inflammatoire [39]. Ces VE contiennent par ailleurs d'importantes quantités du micro-ARN-126, qui favorise la réparation endothéliale vasculaire en ciblant SPREDI (sprouty-related EVHI domain-containing 1) [40], un régulateur négatif de la voie de signalisation du VEGF [41]. Cependant, dans des conditions d'hyperglycémie, l'expression du micro-ARN-126 est réduite dans les vésicules endothéliales, ce qui nuit à la réparation de l'endothélium vasculaire, en raison d'une diminution du ciblage de SPREDI [40]. D'autres travaux ont révélé une expression réduite de ce micro-ARN-126 dans les $V \varepsilon$ circulantes et les $V \varepsilon$ dérivées de progéniteurs endothéliaux de patients atteints de diabète [42]. L'exposition de progéniteurs endothéliaux à ces VE diminue l'expression du récepteur du VEGF, VEGFR2, et la capacité de migration de ces progéniteurs, tout en augmentant l'apoptose et la production de radicaux libres [42].

La raréfaction microvasculaire qui résulte de la mort des cellules endothéliales et d'une angiogenèse myocardique insuffisante, est une manifestation majeure de la maladie cardiovasculaire ischémique induite par le diabète. Au début du diabète, une glycémie élevée entraîne un dysfonctionnement endothélial, favorisant alors cette raréfaction [43]. Des travaux récents ont démontré que les micro-ARN transportés au sein de VE cardiomyocytaires sont étroitement liés aux lésions myocardiques et aux défauts d'angiogenèse liés à la cardiomyopathie diabétique [44]. En utilisant des cardiomyocytes de rats Goto-Kakizaki adultes, un modèle animal couramment employé pour étudier le diabète de type II, Wang et al. [45] ont démontré que le microARN-320 vésiculaire inhibe la prolifération et la migration des cellules endothéliales cardiaques en réduisant 
l'expression des protéines Ets2 (ETS proto-oncogene 2), Hsp20 (heat shock protein 20) et IGF-1 (insulin growth factor 1), ce qui provoque une insuffisance vasculaire au sein du myocarde. Inversement, Garcia et al. [46] ont montré que la privation de glucose conduit à la libération de petites $V \varepsilon$ cardiomyocytaires enrichies en micro-ARN proangiogéniques, tels que le micro-ARN-126-3p et le micro-ARN-23a.

\section{Potentiel thérapeutique des VE et maladies cardiovasculaires}

Ces dernières années, plusieurs études ont mis en évidence la possible utilisation des $V \varepsilon$ comme vecteurs thérapeutiques dans différentes maladies, y compris les maladies cardiovasculaires $[4,47]$. Des études précliniques, réalisées dans des modèles murins d'athérosclérose, ont montré une diminution de la taille des lésions athéromateuses après injection de VE dérivées de cellules endothéliales humaines [41, 48]. L'administration de corps apoptotiques endothéliaux contenant le micro-ARN-126 favorise la sécrétion locale de CXCL12 et l'incorporation de cellules progénitrices exprimant Sca-1 (stem cells antigen-1), ce qui améliore la stabilité de la plaque [41]. Les vésicules transportant le micro-ARN-126, en ciblant SPRED1, stimulent également la ré-endothélialisation après une lésion vasculaire [40]. Dans un modèle murin d'athéroclérose, l'incorporation, dans des cellules musculaires lisses de la plaque, de VE endothéliales enrichies en microARN-143/146 réduit également les lésions athéroscléreuses [48].

Les $V \varepsilon$ présentent également un intérêt pour les thérapies régénératives cardiaques. L'utilisation de thérapies utilisant des cellules souches mésenchymateuses, en particulier, suscite un intérêt considérable. Bien qu'il ait été suggéré que les cellules greffées exercent leur action réparatrice en remplaçant les cellules endommagées, il apparait désormais que le secrétome de ces cellules est en fait responsable de la majorité des bénéfices observés [49]. Cette hypothèse a été confirmée par des travaux montrant que le milieu conditionné des cellules souches, en particulier sa fraction vésiculaire, était capable de réduire la taille de l'infarctus et d'améliorer la fonction cardiaque par la réduction du stress oxydant et l'amélioration de la survie des cardiomyocytes après un infarctus du myocarde. Les petites $V \varepsilon$ des cellules progénitrices cardiaques peuvent par ailleurs orienter les monocytes/ macrophages vers un phénotype réparateur (de type M2) [50]. Les VE dérivées de cellules souches apparaissent ainsi être un substitut intéressant à la greffe de cellules en médecine régénérative après un infarctus du myocarde.

\section{Conclusion et futurs défis}

Les VE jouent un rôle indéniable dans les maladies cardiovasculaires. Des efforts considérables de recherche ont révélé leurs rôles dans la survenue des stades initiaux de maladies telles que l'athérosclérose, ou lors de la survenue d'un infarctus du myocarde. Ces VE sont à la fois de réelles actrices mais également des biomarqueurs de ces maladies. Historiquement, le domaine du cardiovasculaire s'est attaché à étudier le rôle des grandes VE. Le prochain défi de la communauté scientifique est de définir le rôle distinct des différentes sous-populations de VE (grandes ou petites) dans les différents contextes pathologiques. La récente mise en évidence de $V \varepsilon$ d'origine cardiomyocytaire, circulantes, pourrait également permettre d'utiliser ces $V \varepsilon$ comme biomarqueurs spécifiques des atteintes cardiaques. De nombreux travaux restent nécessaires afin d'étudier le devenir de ces $V \varepsilon$ une fois libérées dans la circulation, que ce soit au niveau de leur clairance ou du transfert fonctionnel à distance, afin de préciser leur biodistribution et leurs effets spécifiques. La mise en place de nouveaux modèles expérimentaux, comme le poisson zèbre, pourrait permettre de répondre à ces défis. Identifier et caractériser précisément dans chaque maladie, les mécanismes impliqués dans la libération des VE et dans leur devenir, devraient permettre de développer des stratégies thérapeutiques spécifiques et pertinentes. $\diamond$

\section{SUMMARY}

Extracellular vesicles and cardiovascular diseases

Cardiovascular diseases remain the leading cause of death globally. There is therefore a need to develop new approaches for the treatment and early detection of these ailments. In the past decades, extracellular vesicles ( $\left(\varepsilon V_{s}\right)$ have attracted significant attention as their role in intercellular communication has been brought to light. They have been shown to regulate pathways such as cellular inflammation or angiogenesis, and are therefore involved in key aspects of cardiovascular pathophysiology. Interestingly, EVs appear to have a multifaceted role which depends on their origin and cargo. Though at times deleterious, they have also been proposed as promising diagnostic tools and potential therapeutics. This review highlights recent advances in the role of extracellular vesicles in cardiovascular pathologies. $\diamond$

\section{LIENS D'INTÉRÊT}

Les auteurs déclarent n'avoir aucun lien d'intérêt concernant les données publiées dans cet article.

\section{RÉFÉRENCES}

1. Soehnlein 0 , Libby P. Targeting inflammation in atherosclerosis - from experimental insights to the clinic. Nat Rev Drug Discov $2021 ; 20: 589-610$.

2. Niel G van, D’Angelo $G$, Raposo $G$. Shedding light on the cell biology of extracellular vesicles. Nat Rev Mol Cell Biol 2018 ; 19 : 213-28.

3. Yáñez-Mó M, Siljander PRM, Andreu Z, et al. Biological properties of extracellular vesicles and their physiological functions. J Extracell Vesicles $2015 ; 4: 1-60$.

4. Boulanger $C M$, Loyer $X$, Rautou $P-\varepsilon$, et al. Extracellular vesicles in coronary artery disease. Nat Rev Cardiol $2017 ; 14: 259-72$.

5. Boulanger CM, Scoazec A, Ebrahimian T, et al. Circulating microparticles from patients with myocardial infarction cause endothelial dysfunction. Circulation $2001 ; 104: 2649-52$.

6. Sarlon-Bartoli G, Bennis Y, Lacroix R, et al. Plasmatic level of leukocyte-derived microparticles is associated with unstable plaque in asymptomatic patients with high-grade carotid stenosis. J Am Coll Cardiol 2013; 62 : 1436-41. 


\section{RÉFÉRENCES}

7. Sinning JM, Losch J, Walenta K, et al. Circulating CD31 +/Annexin V + microparticles correlate with cardiovascular outcomes. Eur Heart J 2011 ; 32 : 2034-41.

8. Nozaki T, Sugiyama S, Koga H, et al. Significance of a Multiple Biomarkers Strategy Including Endothelial Dysfunction to Improve Risk Stratification for Cardiovascular Events in Patients at High Risk for Coronary Heart Disease. J Am Coll Cardiol 2009 ; 54 : 601-8.

9. Loyer X, Potteaux S, Vion A, et al. Inhibition of MicroRNA-92a Prevents Endothelial Dysfunction and Atherosclerosis in Mice. Circ Res $2014 ; 114: 434-43$.

10. Zu L, Ren C, Pan B, et al. Endothelial microparticles after antihypertensive and lipid-lowering therapy inhibit the adhesion of monocytes to endothelial cells. Int J Cardiol 2016; $202: 756-9$.

11. Zakharova L, Svetlova M, Fomina AF. T cell exosomes induce cholesterol accumulation in human monocytes via phosphatidylserine receptor. J Cell Physiol 2007 ; 212 : 174-81.

12. Pakala R. Serotonin and thromboxane $A 2$ stimulate platelet-derived microparticle-induced smooth muscle cell proliferation. Cardiovasc Radiat Med $2004 ; 5: 20-6$.

13. Sarkar A, Mitra S, Mehta S, et al. Monocyte derived microvesicles deliver a cell death message via encapsulated caspase-1. PLoS One 2009; 4 .

14. Leroyer AS, Isobe H, Lesèche G, et al. Cellular Origins and Thrombogenic Activity of Microparticles Isolated From Human Atherosclerotic Plaques. J Am Coll Cardiol 2007 ; 49 : 772-7.

15. Mayr M, Grainger D, Mayr U, et al. Proteomics, metabolomics, and immunomics on microparticles derived from human atherosclerotic plaques. Circ Cardiovasc Genet $2009 ; 2$ : 379-88.

16. Tripisciano C, Weiss R, Eichhorn T, et al. Different Potential of Extracellular Vesicles to Support Thrombin Generation: Contributions of Phosphatidylserine, Tissue Factor, and Cellular Origin. Sci Rep 2017 ; $7: 1-11$.

17. Merten $M$, Pakala R, Thiagarajan $P$, et al. Platelet microparticles promote platelet interaction with subendothelial matrix in a glycoprotein Ilb/IIla-dependent mechanism. Circulation 1999; 99 : 2577-82.

18. Srikanthan S, Li W, Silverstein RL, et al. Exosome poly-ubiquitin inhibits platelet activation, downregulates CD36 and inhibits pro-atherothombotic cellular functions. J Thromb Haemost $2014 ; 12$ : 1906-17.

19. Leroyer AS, Rautou PE, Silvestre JS, et al. CD40 Ligand+ Microparticles From Human Atherosclerotic Plaques Stimulate Endothelial Proliferation and Angiogenesis. A Potential Mechanism for Intraplaque Neovascularization. J Am Coll Cardiol 2008 ; 52 : 1302-11.

20. Michel JB, Virmani R, Arbustini $\varepsilon$, et al. Intraplaque haemorrhages as the trigger of plaque vulnerability. Eur Heart J $2011 ; 32$

21. Wadey RM, Connolly KD, Mathew D, et al. Inflammatory adipocyte-derived extracellular vesicles promote leukocyte attachment to vascular endothelial cells. Atherosclerosis $2019 ; 283: 19-27$.

22. Wang $F$, Chen $F$, Shang $Y$, et al. Insulin resistance adipocyte-derived exosomes aggravate atherosclerosis by increasing vasa vasorum angiogenesis in diabetic ApoE -/- mice. Int J Cardiol $2018 ; 265: 181-7$

23. Poisson J, Tanguy M, Davy H, et al. Erythrocyte-derived microvesicles induce arterial spasms in JAK2V617F myeloproliferative neoplasm. J Clin Invest 2020 ; $130: 2630-43$.

24. Camus SM, Moraes JA De, Bonnin P, et al. Circulating cell membrane microparticles transfer heme to endothelial cells and trigger vasoocclusions in sickle cell disease. Blood $2015 ; 125: 3805-14$.

25. Sinning JM, Losch J, Walenta K, et al. Circulating CD31 +/Annexin V + microparticles correlate with cardiovascular outcomes. Eur Heart J 2011 ; $32: 2034-41$.

26. Deddens JC, Vrijsen KR, Colijn JM, et al. Circulating Extracellular Vesicles Contain miRNAs and are Released as Early Biomarkers for Cardiac Injury. J Cardiovasc Transl Res. 2016 ; 9 : 291-301.

27. Rodriguez JA, Orbe J, Saenz-Pipaon G, et al. Selective increase of cardiomyocyte derived extracellular vesicles after experimental myocardial infarction and functional effects on the endothelium. Thromb Res $2018 ; 170: 1-9$.

28. Anselmo A, Frank D, Papa L, et al. Myocardial hypoxic stress mediates functional cardiac extracellular vesicle release. Eur Heart J $2021 ; 42: 2780-92$.

29. Akbar N, Digby JE, Cahill TJ, et al. Endothelium-derived extracellular vesicles promote splenic monocyte mobilization in myocardial infarction. JCl insight 2017 ; 2

30. Loyer X, Zlatanova I, Devue C, et al. Intra-cardiac release of extracellular vesicles shapes inflammation following myocardial infarction short communication. Circ Res 2018 ; 123 : 100-6.

31. Nicolás-Ávila JA, Lechuga-Vieco A V., Esteban-Martínez L, et al. A Network of Macrophages Supports Mitochondrial Homeostasis in the Heart. Cell 2020 ; 183 : 94-109.e23.

32. Bahtiyar G, Gutterman D, Lebovitz H. Heart Failure: a Major Cardiovascular Complication of Diabetes Mellitus. Curr Diab Rep 2016 ; 16 : 116
33. Freeman DW, Noren Hooten $\mathrm{N}$, Eitan $\varepsilon$, et al. Altered Extracellular Vesicle Concentration, Cargo, and Function in Diabetes. Diabetes 2018 ; 67 : 2377 88 .

34. Gan L, Xie D, Liu J, et al. Small Extracellular Microvesicles Mediated Pathological Communications Between Dysfunctional Adipocytes and Cardiomyocytes as a Novel Mechanism Exacerbating Ischemia/Reperfusion Injury in Diabetic Mice. Circulation 2020 ; 141 : 968-83.

35. Carli MF Di, Janisse J, Ager J, et al. Role of chronic hyperglycemia in the pathogenesis of coronary microvascular dysfunction in diabetes. J Am Coll Cardiol $2003 ; 41: 1387-93$

36. Chen J-X, Zeng $\mathrm{H}$, Reese J, et al. Overexpression of angiopoietin-2 impairs myocardial angiogenesis and exacerbates cardiac fibrosis in the diabetic $\mathrm{db} / \mathrm{db}$ mouse model. Am J Physiol Circ Physiol 2012 ; 302 : H1003-12.

37. Caporali A, Meloni M, Völlenkle C, et al. Deregulation of microRNA-503 Contributes to Diabetes Mellitus-Induced Impairment of Endothelial Function and Reparative Angiogenesis After Limb Ischemia. Circulation $2011 ; 123: 282-91$

38. Caporali A, Meloni M, Nailor A, et al. p75NTR-dependent activation of NF- $\kappa B$ regulates microRNA-503 transcription and pericyte-endothelial crosstalk in diabetes after limb ischaemia. Nat Commun 2015; $6: 8024$.

39. Njock MS, Cheng HS, Dang LT, et al. Endothelial cells suppress monocyte activation through secretion of extracellular vesicles containing antiinflammatory microRNAs. Blood $2015 ; 125$ : 3202-12.

40. Jansen F, Yang X, Hoelscher M, et al. Endothelial microparticle-mediated transfer of microRNA-126 promotes vascular endothelial cell repair via spredl and is abrogated in glucose-damaged endothelial microparticles. Circulation 2013 ; 128 : 2026-38.

41. Zernecke A, Bidzhekov K, Noels H, et al. Delivery of microRNA- 126 by apoptotic bodies induces CXCL12-dependent vascular protection. Sci Signal $2009 ; 2$

42. Wu K, Yang $Y$, Zhong $Y$, et al. The effects of microvesicles on endothelial progenitor cells are compromised in type 2 diabetic patients via downregulation of the miR-126/VEGFR2 pathway. Am J Physiol Metab 2016 310 : ع828-37.

43. Nakagami $H$, Kaneda $Y$, Ogihara $T$, et al. Endothelial Dysfunction in Hyperglycemia as a Trigger of Atherosclerosis. Curr Diabetes Rev $2005 ; 1$ : 59-63.

44. Nandi SS, Mishra PK. Targeting miRNA for Therapy of Juvenile and Adult Diabetic Cardiomyopathy. Advances in experimental medicine and biology. Adv Exp Med Biol 2018 : 47-59.

45. Wang $X$, Huang $W$, Liu G, et al. Cardiomyocytes mediate anti-angiogenesis in type 2 diabetic rats through the exosomal transfer of miR-320 into endothelial cells. J Mol Cell Cardiol $2014 ; 74$ : 139-50.

46. Garcia NA, Ontoria-Oviedo I, González-King H, et al. Glucose Starvation in Cardiomyocytes Enhances Exosome Secretion and Promotes Angiogenesis in Endothelial Cells. PLoS One 2015 ; 10 : e0138849.

47. Wiklander OPB, Brennan M, Lötvall J, et al. Advances in therapeutic applications of extracellular vesicles. Sci Transl Med 2019; 11:8521.

48. Hergenreider $\varepsilon$, Heydt $S$, Tréguer $K$, et al. Atheroprotective communication between endothelial cells and smooth muscle cells through miRNAs. Nat Cell Biol 2012 ; 14 : 249-56.

49. Lai RC, Chen TS, Lim SK. Mesenchymal stem cell exosome: A novel stem cellbased therapy for cardiovascular disease. Regen Med 2011 ; $6: 481-92$.

50. Pezzana C, Agnely F, Bochot A, et al. Extracellular Vesicles and Biomaterial Design: New Therapies for Cardiac Repair. Trends Mol Med 2021 ; 27 : 231-47.

\section{Retrouvez toutes les Actualités de la Myologie sur les sites de}

la Société Française de Myologie

www.sfmyologie.org la filière de santé neuromusculaire FILNEMUS

www.filnemus.fr
TIRÉS À PART

$X$. Loyer 